Introduction: The Diet Quality Index (DQI) is a tool that assesses diet quality in terms of compliance with the Flemish Food-based Dietary Guidelines. The objective was to investigate the correlation between the DQI score of healthy European adolescents and their body composition and socio-economic status.

Method: The study population aged $12 \cdot 5-17 \cdot 5$ years, consisted of 3002 healthy European adolescents (1582 females) from the HELENA Study. Information on dietary intake was obtained via two, non-consecutive, self-reported $24 \mathrm{~h}$ recalls. DQI scores were calculated for all respondents. Partial Pearson correlations $(r)$ were calculated, adjusting for the influence of sex, age and Tanner stage.

Results: Mean DQI for boys and girls was, respectively, $51 \cdot 23(\mathrm{sD} 18 \cdot 31)$ and $55 \cdot 88(\mathrm{sD} 16 \cdot 69)(P<0 \cdot 001)$. Low but significant positive correlations were found between DQI and BMI $Z$-scores $(r=0.051 ; P=0 \cdot 006)$. In separate analyses for both sexes, correlations were stronger in the female group. Similar results were found for skin folds, hip and waist circumference. Correlations with blood pressure were negative; however, only borderline significance was found for the systolic values $(r=-0 \cdot 037$; $P=0 \cdot 044)$. Strong correlations were found with the educational level of both mother $(r=0 \cdot 22 ; P<0 \cdot 001)$ and father $(r=0 \cdot 17 ; P<0 \cdot 001)$.

Discussion: Higher DQI scores were associated with lower systolic blood pressure and higher educational level of both parents, confirming the importance of socio-economic status on health and dietary habits. The positive correlations with BMI Z-scores and markers of body composition might be attributed to (i) a tendency of under-reporting by overweight people or (ii) following a healthier diet to loose weight.

\title{
15 - Dietary under-reporting by overweight and obese adolescents: results from the HELENA Study
}

\author{
I Huybrechts ${ }^{1}$, E Grammatikaki ${ }^{2},{ }^{2}$ Kersting ${ }^{3}, \mathrm{~K}$ Vyncke ${ }^{1}, \mathrm{M} \mathrm{Cuenca}^{3}$, \\ M Gonzalez-Gross ${ }^{3}$, F Gottrand ${ }^{4}$, LA Moreno', D Molnar' ${ }^{6}$, L Hallstrom ${ }^{7}$, J Dallongeville ${ }^{4}$,

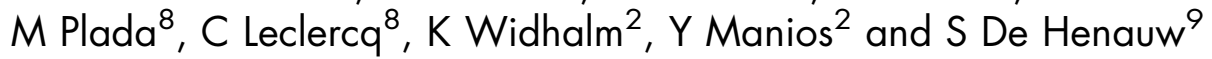 \\ ${ }^{1}$ Department of Public Health, Ghent University, Ghent, Belgium: ${ }^{2}$ Department of Nutrition and Dietetics, Harokopio \\ University, Athens, Greece: ${ }^{3}$ Research Institute of Child Nutrition, Rheinische Friedrich-Wilhelms Universität Bonn, \\ Heinstueck 11, Dortmund, Germany: ${ }^{4}$ Institut Pasteur de Lille, Lille, France: ${ }^{5}$ Escuela Universitaria de Ciencias de la \\ Salud, Universidad de Zaragoza, Zaragoza, Spain: ${ }^{6}$ University of Pecs, Pecs, Hungary: 7 Unit for Preventive Nutrition, \\ Department of Bioscience and Nutrition, Karolinska Institutet, Huddinge, Sweden: ${ }^{8}$ Preventive Medicine \& Nutrition Unit, \\ University of Crete, Heraklion, Crete, Greece: ${ }^{9}$ Department of Health, Ghent University, Ghent, Belgium
}

Aim: To examine under-reporting of energy intakes (EI) in relation to overweight/obesity among adolescents.

Design: Two self-administered computerized $24 \mathrm{~h}$ recalls were used in 2330 adolescents aged $12 \cdot 5-17 \cdot 5$ years across eight EU countries (Austria, Belgium, France, Germany, Greece, Italy, Spain and Sweden). Height, weight and circumferences were measured as body composition indicators. International BMI cut-offs of Cole/IOTF were used to assess overweight/obesity prevalence. Slaughter formula was used to calculate body fat percentage (BF\%). BMR was calculated from age-specific FAO/WHO/UNU equations and under-reporting via Goldberg cut-offs for EI/BMR.

Results: The degree of under-reporting was higher for the second 24 h-recall administration than for the first $(23 \% v$. $19 \cdot 8 \%)$ and higher among girls than among boys (24.3\% $v$. $20.5 \%$ ). When considering mean energy intakes (EI) of the $2 \mathrm{~d}, 22.5 \%$ of the adolescents were categorized as underreporter. Prevalence of under-reporting was significantly higher in overweight and obese adolescents than in normal/ underweight adolescents (43.9\% and $50 \cdot 8 \%$ v. $16 \cdot 7 \%$, respectively). BMI $Z$-scores and $\mathrm{BF} \%$ were negatively correlated with EI/BMR $(-0 \cdot 35$ and $-0 \cdot 38)$.

Discussion: Although under-reporting appeared in all BMI categories, the prevalence increased with increasing BMI category. These results confirm the general assumption that overweight/obese people tend to under-report more frequently than normal weight people. Possible hypotheses could be that (i) overweight/obese people are on a diet and truly reporting low EI or (ii) they are more prone to social desirable answers. These results may have implications for the interpretation of studies of diet and comorbidities related to obesity among adolescents.

Funding: The HELENA Study was carried out with the financial support of the European Community Sixth RTD Framework Programme (Contract FOOD-CT-2005007034). The content of this article reflects only the authors' views, and the European Community is not liable for any use that may be made of the information contained therein from rural area in South-East of Poland. 